\title{
Transforming pollen grains from an allergy causing material into a biomaterial for oral vaccination
}

\author{
Harvinder Singh Gill, PhD
}

For many people, the words 'pollen grains' are likely to conjure an image of a runny nose and itchy eyes. This is because pollens can cause respiratory allergies. Looking beyond this allergic response, scientists are harnessing the toughness of the pollen shells to develop them into carriers for oral drug and vaccine delivery. ${ }^{1-4}$ Pollen grains exist in nature not to cause allergies but to transport the male gamete for fertilization to the ovary, which is located on the flower. To perform this function, pollens often have to traverse long distances and can experience a dramatic change in temperature and humidity due to weather fluctuations. To protect the male gamete against these thermal and hydration forces, pollens have an outer solid wall made from a material called the sporopollenin. This wall is mechanically tough and is resistant to chemical degradation. ${ }^{5}$ The pollen wall by itself is rather benign and does not cause allergies. The allergic response to pollen exposure is caused by the proteins and biomolecules that reside on or within the walls of the pollens. It is precisely due to this reason that the skin prick test for pollen allergies is done by using pollen extracts (proteins and biomolecules extracted from intact pollens) and not with intact pollen shells.

The pollen shell with its tough outer wall has recently gained attention as a potential microcarrier for the delivery of drugs and vaccines through the oral route. The premise behind this is that the toughness and durability of the pollen shell can allow it to stay unharmed in the acid and the enzyme rich environment of the stomach, allowing it to carry the drug payload safely into the intestine where it can be released for absorption. To use pollens for biomedical applications, it is imperative that their potentially allergy causing biomolecules are

Corresponding author: Harvinder Gill

Contact Information: Harvinder.gill@ttu.edu

DOI: 10.12746/swrccc.v7i27.510 removed to prevent accidental allergy sensitization. My laboratory at Texas Tech University has made a significant contribution to the processing of raw pollens to obtain clean pollens. There is a well established chemical treatment protocol to treat Lycopodium clavatum spores to obtain spores that are devoid of proteins and other biomolecules; however, when this process is applied to pollens of other species, it fails. We have recently developed a new chemical treatment process, which is broadly applicable to pollens of different species. ${ }^{6}$ Using this process, we have successfully produced clean pollens from ragweed, sunflower, black alder, and lamb's quarter (Figure 1).

Oral vaccination is painless, child friendly, and convenient to administer, yet it remains limited to few commercial vaccines; the majority of the vaccines are still administered via the intramuscular route. My group has been studying the potential of pollen grains for oral vaccination for many years. The in vivo proof-of-concept for oral vaccination with pollens was first demonstrated by
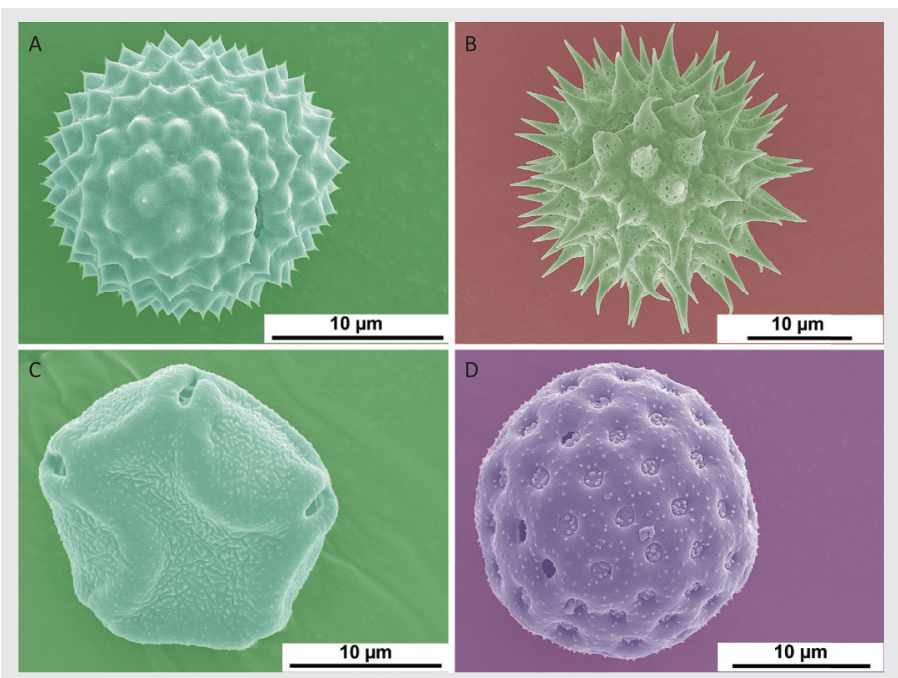

Figure 1. Scanning electron micrographs of different pollen grains after chemical treatment. (A) Ragweed, (B) sunflower, (C) alder black, and (D) lambs quarter. 
my group. We showed that when a model protein called ovalbumin was filled in the clean spores of Lycopodium clavatum and fed to mice, both systemic IgG and intestinal mucosal $\lg \mathrm{A}$ antibody responses specific to ovalbumin could be generated. ${ }^{2}$ Of note, the response persisted for up to seven months after vaccination. In a subsequent study we used clean ragweed pollen shells and demonstrated that ragweed pollens also have the ability to act as microcapsules for oral vaccination. ${ }^{7}$ In this study we again observed, long-lived systemic and mucosal antibody responses in mice. When bone marrow cells of immunized mice were cultured ex vivo, antibody secretion was observed suggesting that oral immunization had led to the development of antibody secreting plasma cells. It was seen that IgE antibodies specific to ovalbumin were not generated in the vaccinated mice, suggesting that oral vaccination with pollens does not cause allergic sensitization.

To gather a mechanistic understanding and to evaluate biocompatibility, we have recently performed multiple in vitro and in vivo experiments. ${ }^{8}$ Using Caco-2 human intestinal epithelial cells, we have shown that clean ragweed pollens are biocompatible and that they do not affect cellular permeability. Interestingly, IL-6, IL-8, and MCP-1 (monocyte chemoattractant protein-1) were also secreted by the Caco-2 cells. These cytokines are known to be potent recruiters and activators of immune cells, such as the neutrophils and macrophages. Furthermore, when clean ragweed pollens were incubated with bone marrow derived macrophages and dendritic cells, a higher expression of the pro-inflammatory cytokines, such as TNF- $\alpha$ and IL-1 $\beta$, was observed. The macrophage cells were seen to phagocytose not just ragweed but other pollens as well. Figure 2 shows multiple macrophage cells trying to phagocytose sunflower pollens. The dendritic cells also showed an upregulation of CD40, MHC-II, CD80, and CD86 suggesting a role for clean pollen shells in activating dendritic cells. These experiments suggested that the ragweed pollens have an adjuvant-like function and can activate the immune system. It was also seen that the protein (ovalbumin) formulated with the clean pollen shell gets adsorbed on the pollen wall. This protein could not be detached despite thorough washing of the pollens. When clean ragweed pollens were fed to mice and the intestinal wall was examined

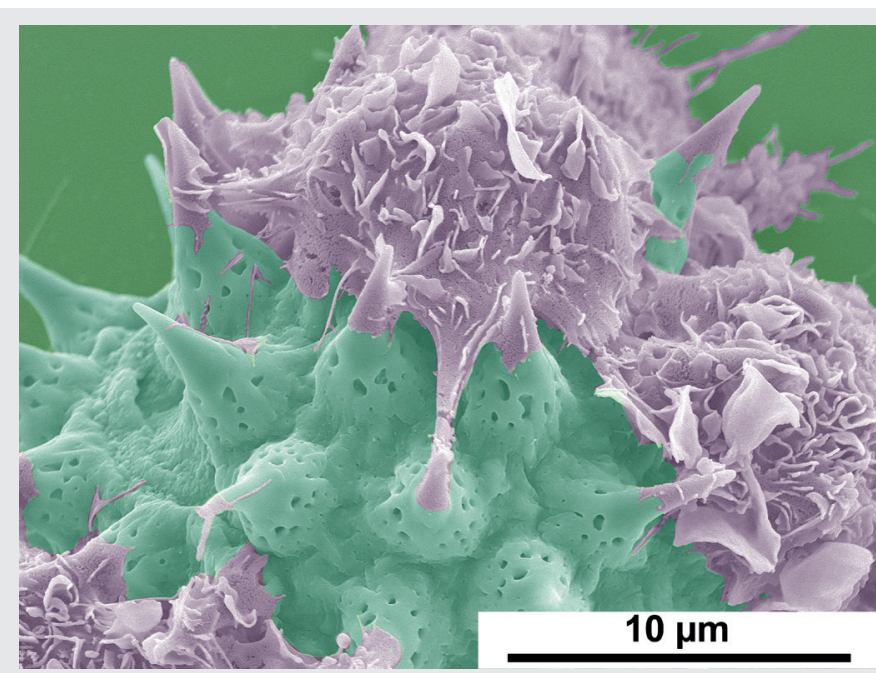

Figure 2. Scanning electron micrograph of mouse bone marrow derived macrophages phagocytosing a sunflower pollen grain.

24 hours later, pollens could be seen in the subepithelial space suggesting uptake of the pollens after oral ingestion. Based on this study, we proposed that pollens acted both as an adjuvant and a transporter of the adsorbed protein, and this combined action helped generate a good immune response.

From a safety perspective, pollens have a history of being used as a health food in the form of bee pollen. While this offers some anecdotal evidence that pollens are safe for oral consumption, more controlled human studies are required to document the safety of pollens. These studies can then lay the foundation of testing pollen grain-based oral vaccine formulations in humans in the hope of developing edible vaccines.

Keywords: Pollen grains, vaccination, allergy

From: Department of Chemical Engineering, Texas Tech University, Lubbock, Texas

Submitted: $12 / 27 / 2018$

Conflicts of interest: none

This work is licensed under a Creative Commons Attribution-ShareAlike 4.0 International License. 


\section{REFERENCES}

1. Akyuz L, Sargin I, Kaya M, Ceter T, et al. A new pollenderived microcarrier for pantoprazole delivery. Materials science\& engineeringC,Materials forbiologicalapplications2017; 71:937-42.

2. Atwe SU, Ma Y, Gill HS. Pollen grains for oral vaccination. J Control Release 2014;194:45-52.

3. Diego-Taboada A, Beckett ST, Atkin SL, et al. Hollow pollen shells to enhance drug delivery. Pharmaceutics 2014;6:80-96.

4. Lale SV, Gill HS. Pollen grains as a novel microcarrier for oral delivery of proteins. Int J Pharm 2018;552:352-9.
5. Qu Z, Meredith JC. The atypically high modulus of pollen exine. J Royal Society, Interface 2018;15.

6. Gonzalez-Cruz P, Uddin MJ, Atwe SU, et al. Chemical treatment method for obtaining clean and intact pollen shells of different species. ACS Biomaterials Science \& Engineering 2018;4:2319-29.

7. Uddin MJ, Gill HS. From allergen to oral vaccine carrier: A new face of ragweed pollen. Int J Pharm 2018;545:286-94.

8. Uddin MJ, Gill HS. Ragweed pollen as an oral vaccine delivery system: Mechanistic insights. J Control Release 2017;268: 416-26. 